\title{
Comparison Between the Size Distributions Determined on Random Plane Sections and Projected Shadows of Non-Spherical Particles
}

\author{
Yoshimoto Wanibe, Takashi Itoh \\ Yoshiharu Terada and Takashi Ohara
}

Dept. Materials Processing Engineering, Nagoya University, Furo-cho Chikusa-ku Nagoya 464-01.

Received June 3, 1993

\begin{abstract}
From measurements made on random sections of non-spherical objects, the shape functions have been statistically determined and the size distributions have been able to be stereologically estimated. Then the distributions obtained as histograms have been summarized by using the proposed compound log normal law. The results are quantitatively compared with the other corresponding ones which have been procured from the measurements made on the projected shadows of the identical objects. According to discussion on the effect of the shape functions on the size distributions, mean values of the size estimated on the usual assumption of spherical objects contain the relative errors amounting to $10 \sim 40 \%$ at the circularity of even less than 0.98 .
\end{abstract}

\section{Introduction}

When the authors have proposed a two dimensional diagram of projected area diameters (or Heywood's diameters) and circularities and the simultaneous analysis of both the distributions in sizes and shapes of powder particles, the measurements have been made on

I. shadowgraphs of dispersed powder particles, ${ }^{1)}$ and

II. random plane sections of the particles mounted with resin. ${ }^{2)}$

In the former measurement, there is a merit that the physical images of particles are easily understandable except in the case of extremeness in shapes, such as flakes, aciculars, etc., but a demerit that the application is limited to examination of only discrete particles like powders. In the latter, the application may be extended much beyond the above boundary to the investigation of microstructures and pores in even consolidated bodies. However, restrictions are imposed on the conditions when three dimensional particles must be estimated from measurements made on the random plane sections.

There have been the other studies ${ }^{3,4)}$ which aim at improving the practical applicability of the stereology to determine the size distribution of non-spherical objects from measurements made only on the random plane sections. Two kinds of measurement have been carried out

I. to determine dimensionless shape functions (a kind of the statistical mean shape factors) of particles, and

II. to obtain distributions of the unidirectional (or statistical) maximum diameters, the Krummbein's diameters.

The determinable distributions are of the maximum diameters of objects based on the statistic mean shape.

Then it is interesting to compare these results with those of the area diameters, which have been determined from the measurements 
made on both the shadow graphs ${ }^{1)}$ and the random plane sections ${ }^{2)}$ of the identical powders.

(a)

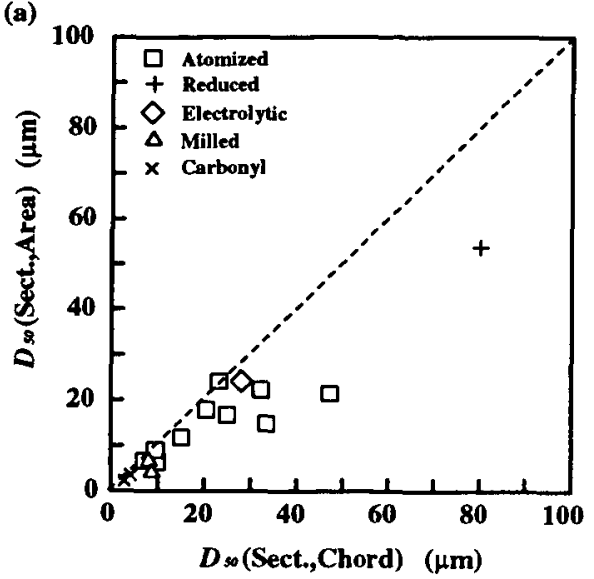

(b)

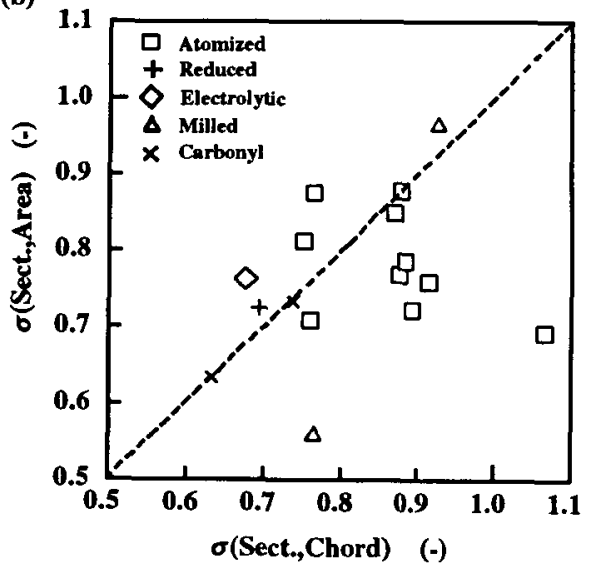

Fig.1 Comparison between the size distributions of the chord diameters and the sectional area ones; (a) shows the relation between the mean particle size diameters and (b) the relation between the standard deviations.

\section{Measurements and Analysis}

All the details of the measurement and the analysis, such as the powders measured, the preparation of samples, the apparatus and procedures for measurements and statistical examinations, have been already described in the previous works. ${ }^{1-4}$ )

\section{Discussion}

3.1 Chord Diameters and Area Ones Measured on Sections
As for the sectional figures which are formed by intersecting the powder particles with a plane, two kinds of expression have been given to the sizes of the particles; the one is of the unidirectional maximum diameters of the figures,") while the other has been of the area diameters ${ }^{2}$. In order to examine the difference in choice of the diameters, Figure 1 shows the comparison between both the distributions of the chord diameters and the area ones, which are summarized according to the usual $\log$ normal formula. Hereinafter, scales in figures mutually concerned are unified as much as possible for convenience of quantitative comparison, and all the notation used are appended in the previous report. ${ }^{3)}$ The chord diameters are larger than the area ones as to the mean values, the differences of which vary with the particle shapes. As for the geometric standard deviations, there is no obvious relation between both the measurements, scattering according to the shapes, even though the former deviations may have tendency of being a little larger than the latter ones.

\subsection{Stereological Chord Diameters and Pro- jected Area Ones}

There have been the other measurement of the area diameters determined differently on the shadow graphs of individual particles for the identical powders. ${ }^{1)}$ Then these size distributions of the area diameters are compared with those of the maximum chord diameters which have been stereologically estimated. The results are shown in Figure 2. It is natural that the mean values of the stereological chord lengths should be in principle larger than those of the area diameters. The figure also shows that the standard deviations of both the distributions have a good agreement to each other.

\subsection{Effect of Shapes on Size Distributions}

Interesting is difference between both the 
(a)

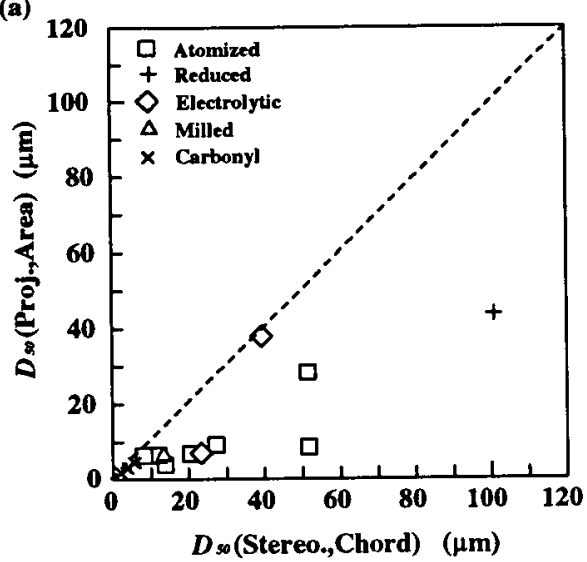

(b)

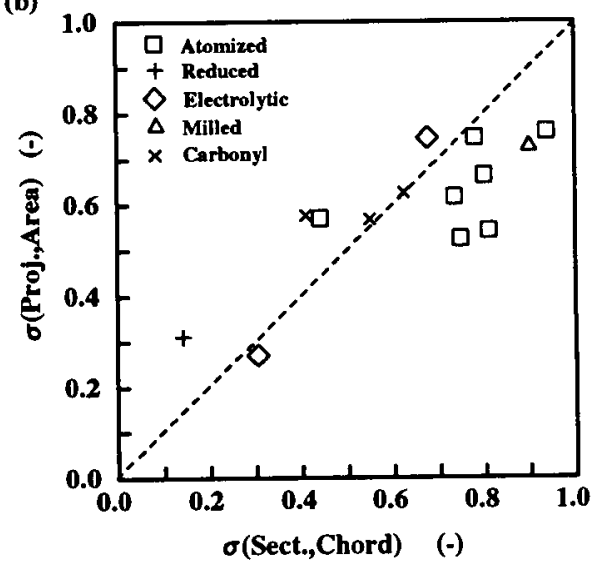

Fig.2 Comparison between the size distributions of the chord diameters and the projected area ones; (a) shows the relation between the mean particle size diameters and (b) the relation between the standard deviations.

size distributions estimated by using the dimensionless shape function and an imaginary assumption of spherical shape (as has been done in most conventional analyses). By using the dimensionless function of a spherical particle like Figure 3, each imaginary distribution and its mean diameter can be calculated from the same data through the same algorithm for all the samples measured. In order to assess the efficacy of the function, Figure 4 shows the plots between the circularity based on the shape function and the relative difference in both the mean diameters, one of which estimated with the actual shape function and the

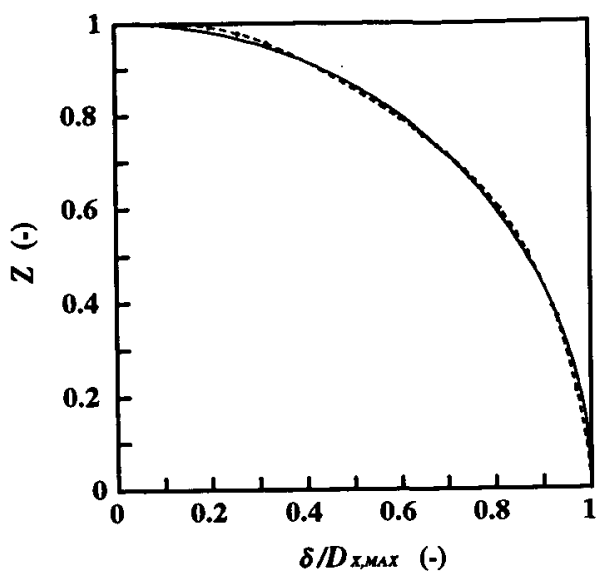

Fig.3 Dimensionless figure (solid line) and function (broken line) of a sphere.

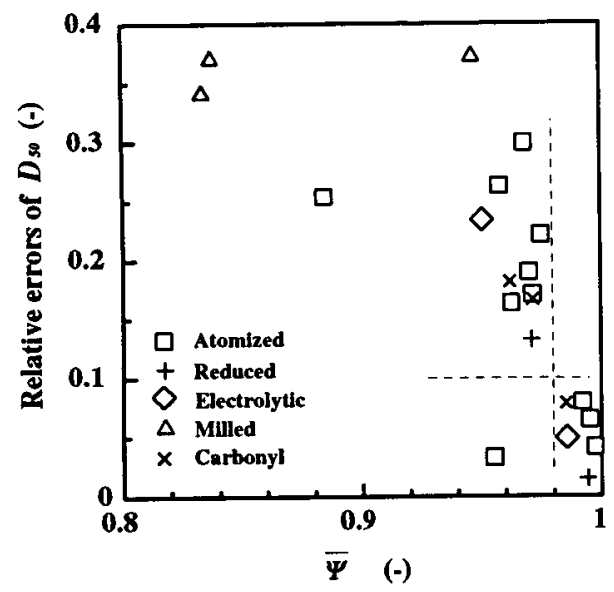

Fig.4 Efficacy of the dimensionless function.

other of which with the spherical assumption. It reveals that if the mean diameter is estimated without the shape function, the relative error amounts to $10 \sim 40 \%$ at the circularity of even less than 0.98 .

\section{Conclusion}

As for the sectional figures which are formed by intersecting the non-spherical particles with a plane, two kinds of expression have been given to the sizes of the particles; the one of the unidirectional maximum diameters $^{4)}$ and the other of the area diameters ${ }^{2}$. The size distribution which has been able to be 
stereologically estimated from the figures is of the maximum diameters and based on the statistic mean shape. For the identical powders, there has been also the distribution of the area diameters based on the shadow graph of individual particles. ${ }^{1)}$ The quantitative comparison among the above results and the discussion on the effect of the shape on the size distribution are summarized to be:

1. The mean values of the stereological chord lengths are larger than those of the area diameters, but both the standard deviations of the distributions have a good agreement to each other.

2. Popular assumption of spherical particles may lead a false estimation of the mean diameter over a size distribution with a relative error of $10 \sim 40 \%$ at the circularity of even less than 0.98 .

The latter finding is quite important in quantitative characterization, not only of discrete particles in powders, but also of particles and pores even in solid structures of multiphases.

\section{Acknowledgments}

The authors are greatly indebted to Kobe Steel Co. and Kawasaki Steel Co. for the kind provision of the powders used. The numerical calculations and part of the preparation of the figures have been performed with a FACOM M-1800 machine in the Nagoya University Computation Center.

\section{References}

1. T. Itoh and Y. Wanibe: Powder Metall., 34(1991), 126.

2. T. Itoh and Y. Wanibe: 5. Europ. Symp. 'Particle Characterization' (467. Event of Europ. Fed. Chem. Engg.), Nuernberg, Germany, March 1982; Ahsts., 31 \& Preprints 1, 243; Particle Characterisation, pending publication.

3. Y. Wanibe, T. Itoh, Y. Terada and T. Ohara: J. Japan Soc Powder and Powder Metallurgy, 40(1993), 1109.

4. Y. Wanibe, T. Itoh, Y. Terada and T. Ohara: J. Japan Soc Powder and Powder Metallurgy, 40(1993), 1113. 